\title{
Capecitabine Pattern of Usage, Rate of Febrile Neutropaenia and Treatment Related Death in Asian Cancer Patients in Clinical Practice
}

\author{
Vincent Chee Ee Phua ${ }^{1 *}$, Wei Quan Wong², Pei Lin Tan³, Anita Zarina Bustam¹, \\ Marniza Saad ${ }^{1}$, Adlinda Alip ${ }^{1}$, Wan Zamaniah Wan Ishak ${ }^{1}$
}

\begin{abstract}
Background: Oral capecitabine is increasingly replacing intravenous 5 -fluorouracil in many chemotherapy regimens. However, data on the risk of febrile neutropaenia (FN) and treatment related death (TRD) with the drug remain sparse outside of clinical trial settings despite its widespread usage. This study aimed to determine these rates in a large cohort of patients treated in the University of Malaya Medical Centre (UMMC). Materials and Methods: We reviewed the clinical notes of all patients prescribed with oral capecitabine chemotherapy for any tumour sites in University Malaya Medical Centre (UMMC) from $1^{\text {st }}$ January 2009 till $31^{\text {st }}$ June 2010. Information collected included patient demographics, histopathological features, treatment received including the different chemotherapy regimens and intent of treatment whether the chemotherapy was given for neoadjuvant, concurrent with radiation, adjuvant or palliative intent. The aim of this study is to establish the pattern of usage, FN and TRD rates with capecitabine in clinical practice outside of clinical trial setting. FN is defined as an oral temperature $>38.5^{\circ} \mathrm{C}$ or two consecutive readings of $>38.0^{\circ} \mathrm{C}$ for 2 hours and an absolute neutrophil count $<0.5 \mathrm{x}$ $10^{\%} / \mathrm{L}$, or expected to fall below $0.5 \times 10^{9} / \mathrm{L}$ (de Naurois et al., 2010). Treatment related death was defined as death occurring during or within 30 days of last chemotherapy treatment. Results: Between $1^{\text {st }}$ January 2009 and $30^{\text {th }}$ June 2010, 274 patients were treated with capecitabine chemotherapy in UMMC. The mean age was 58 years (range 22 to 82 years). Capecitabine was used in 14 different tumour sites with the colorectal site predominating with a total of 128 cases $(46.7 \%)$, followed by breast cancer $(35.8 \%)$. Capecitabine was most commonly used in the palliative setting accounting for $63.9 \%$ of the cases, followed by the adjuvant setting $(19.7 \%)$. The most common regimen was single agent capecitabine with 129 cases $(47.1 \%)$. The other common regimens were XELOX $(21.5 \%)$ and ECX $(10.2 \%)$. The main result of this study showed an overall FN rate of $2.2 \%(6 / 274)$. The overall TRD rate was 5.1\% (14/274). The FN rate for the single agent capecitabine regimen was $1.6 \%(2 / 129)$ and the TRD rate was $5.4 \%$ (7/129). All the TRDs were with single agent capecitabine regimen were used for palliative intent. Conclusions: Oral capecitabine is used widely in clinical practice in a myriad of tumour sites and bears a low risk of febrile neutropaenia. However, capecitabine like any other intravenous chemotherapeutic agent carries a significant risk of treatment related death.
\end{abstract}

Keywords: Capecitabine - chemotherapy - febrile neutropaenia - treatment related death

Asian Pac J Cancer Prev, 16 (4), 1449-1453

\section{Introduction}

Capecitabine $\left(\mathrm{Xeloda}^{\circledR}\right)$ is an oral prodrug that is converted to 5-fluorouracil (5-FU) in a three-step enzymatic process, the final stage of which is mediated by thymidine phosphorylase (TP). Significantly higher TP activity has been recorded in a number of human tumour tissues (including colorectal, breast, stomach, cervix, uterus, ovary, kidney, bladder, and thyroid), compared with normal tissue adjacent to the tumour, allowing capecitabine to generate 5-FU preferentially at the tumour site (Miwa et al., 1998). Widespread usage of this drug has taken hold in this country due to its easy administration obviating the need for day care or long hospital admissions or the risks associated with chemoport usage for long infusional regimens using 5-FU. Randomized trials have shown acceptable efficacy and safety profile in breast, colorectal and upper gastrointestinal tumours (Van Custem et al., 2001; Cassidy et al., 2008; Cunningham et al., 2008; Kang et al., 2009; Comella et al., 2009; Okines et al., 2009;

${ }^{1}$ Clinical Oncology Unit, Faculty of Medicine, ${ }^{3}$ Pharmacology Department, University Malaya Medical Centre, University of

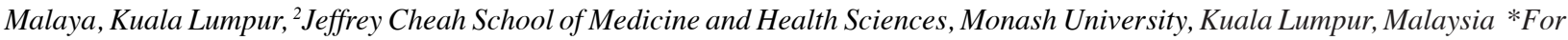
correspondence: vince_phua@yahoo.com 
Sym et al., 2010; Cassidy et al., 2011; Stockler et al., 2011; Ducreux et al., 2011; Wadel et al., 2012). Meanwhile, in other less common tumour sites phase II trials have evaluated its efficacy including pancreatic tumour (Xiong et al., 2008; Choi et al., 2012), nasopharyngeal carcinoma (Chua et al., 2003; Chua 2008; Ciuleanu et al., 2008; Li et al., 2008; Chua et al., 2012), head and neck cancers (Martinez-Trufero et al., 2010), ovarian cancer (Vasey et al., 2003) and cervical cancer (Jenkins et al., 2005). However, there is a paucity of data regarding its pattern of usage in Asian countries, rate of febrile neutropaenia (FN) and treatment related death (TRD). A recent paper published by researchers in Hong Kong on the efficacy of adjuvant XELOX regimen for colorectal cancer (Xeloda plus oxaliplatin) reported good efficacy and tolerability but did not report the rates of FN and TRD (Chiu et al., 2014). Another publication on capecitabine usage for elderly patients with metastatic colorectal cancer revealed no FN and TRD but there were only 36 patients in this study (Varol et al., 2014). The same group of researchers reported on the efficacy of XELOX regimen in metastatic pancreatic cancer resistant to gemcitabine treatment on 47 patients but did not specifically report on the FN and TRD rates (Bayoglu et al., 2014). Capecitabine was also tested in a small study comprising 54 patients with Her-2 negative advanced gastric cancer in combination with modified dose docetaxel and cisplatin. This retrospective cohort study reported good efficacy and FN rate was $3.7 \%$ (2/54) but TRD rate was not reported. However, they did report no death resulted from the two cases of FN (Bilici et al., 2014).

Despite capecitabine's widespread adoption into many common chemotherapy regimens for a myriad of tumour sites, there remains a paucity of clinical data in clinical practice outside of clinical trial regarding the rate of FN and TRD. Patients and even clinicians often assume that an oral drug is less toxic compared to its intravenous counterpart despite both being chemotherapeutic agents with risks of FNs and TRDs. It is in this light that we embarked on this study to evaluate the rate of $\mathrm{FN}$ and TRD with capecitabine and also to determine the range of its usage in different tumour sites in our institution.

\section{Materials and Methods}

We reviewed the clinical notes of all patients prescribed with oral capecitabine chemotherapy for any tumour sites in University Malaya Medical Centre (UMMC) from 1st January 2009 till 31 $1^{\text {st }}$ June 2010. Information collected included patient demographics, histopathological features, treatment received including the different chemotherapy regimens and intent of treatment whether the chemotherapy was given for neoadjuvant, concurrent with radiation, adjuvant or palliative intent. The aim of this study is to establish the pattern of capecitabine usage, FN and TRD rate with capecitabine in clinical practice outside of clinical trial setting. FN is defined as an oral temperature $>38.5^{\circ} \mathrm{C}$ or two consecutive readings of $>38.0^{\circ} \mathrm{C}$ for 2 hours and an absolute neutrophil count $<0.5$ x $10^{9} / \mathrm{L}$, or expected to fall below $0.5 \times 10^{9} / \mathrm{L}$ (de Naurois et al., 2010). Treatment related death is defined as death occurring during or within 30 days of last chemotherapy treatment as a consequence of the chemotherapy treatment.

\section{Results}

Between $1^{\text {st }}$ January 2009 and $30^{\text {th }}$ June 2010, 274 patients were treated with capecitabine chemotherapy in UMMC. Baseline characteristics including clinicopathological and treatment modalities utilized are summarized in Table 1. The mean age of patients in this study was 58 years (range 22 to 82 years). There were 25 patients $(1.6 \%)$ older than 70 years in this study. Capecitabine was used in 14 different tumour sites with

Table 1. The Clinicopathological Characteristics of the 274 Patients Treated with Capecitabine in UMMC

\begin{tabular}{|c|c|c|}
\hline Items & $\begin{array}{c}\text { No. of } \\
\text { patients }\end{array}$ & $\%$ \\
\hline \multicolumn{3}{|l|}{ Age } \\
\hline Up to 50 years & 61 & 46.8 \\
\hline $51-69$ years & 188 & 51.6 \\
\hline 70 years and above & 25 & 1.6 \\
\hline \multicolumn{3}{|l|}{ Gender } \\
\hline Male & 93 & 31.7 \\
\hline Female & 181 & 53.2 \\
\hline \multicolumn{3}{|l|}{ Primary Site } \\
\hline Breast & 98 & 35.8 \\
\hline Colon & 63 & 22.9 \\
\hline Rectum & 50 & 18.2 \\
\hline Rectosigmoid & 14 & 5.1 \\
\hline Gastric & 26 & 9.5 \\
\hline Oesophagus & 8 & 2.9 \\
\hline Appendix & 3 & 1.1 \\
\hline Anal & 1 & 0.4 \\
\hline Gallbladder & 1 & 0.4 \\
\hline Pancreas & 1 & 0.4 \\
\hline Parotid & 1 & 0.4 \\
\hline Antrum & 1 & 0.4 \\
\hline Nasopharyngeal carcinoma & 2 & 0.7 \\
\hline Unknown Primary & 5 & 1.8 \\
\hline \multicolumn{3}{|l|}{ AJCC Stage } \\
\hline 1 & 1 & 5.9 \\
\hline 2 & 31 & 49.9 \\
\hline 3 & 64 & 11.6 \\
\hline 4 & 177 & 11.8 \\
\hline \multicolumn{3}{|l|}{ Chemotherapy objective } \\
\hline Neoadjuvant & 5 & 1.8 \\
\hline Concurrent chemoirradiation & 18 & 6.6 \\
\hline Concurrent chemoirradiation then adjuvant & 22 & 8 \\
\hline Adjuvant & 54 & 19.7 \\
\hline Palliative & 175 & 63.9 \\
\hline \multicolumn{3}{|l|}{ Chemotherapy Regimen } \\
\hline XELODA ALONE & 129 & 47.1 \\
\hline XELODA + LAPATINIB & 17 & 6.2 \\
\hline XELODA + MITOMYCIN C & 6 & 2.2 \\
\hline XELODA + HERCEPTIN & 3 & 1.1 \\
\hline XELODA + VINORELBINE & 5 & 1.8 \\
\hline XELOX & 59 & 21.5 \\
\hline XELOX + AVASTIN & 5 & 1.8 \\
\hline XELIRI & 4 & 1.5 \\
\hline XELIRI + AVASTIN & 8 & 2.9 \\
\hline ECX & 28 & 10.2 \\
\hline EOX & 2 & 0.7 \\
\hline XELODA + DOCETAXEL & 3 & 1.1 \\
\hline OTHERS & 5 & 1.8 \\
\hline
\end{tabular}


the colorectal site predominating with a total of 128 cases $(46.7 \%)$ followed by breast cancer $(35.8 \%)$. Upper gastrointestinal cancers accounted for 34 cases $(12.4 \%)$. Capecitabine was most commonly used in the palliative setting accounting for $63.9 \%$ of the cases followed by the adjuvant setting (19.7\%). The most common regimen was single agent capecitabine with 129 cases $(47.1 \%)$. The other common regimens were XELOX regimen (21.5\%) and ECX regimen (10.2\%).

The main result of this study showed an overall FN rate of $2.2 \%(6 / 274)$. The overall TRD rate was $5.1 \%(14 / 274)$. Thirteen of these patients were treated with palliative intent and one was treated for neoadjuvant chemotherapy to be followed by definitive surgery. This patient had stage 3 gastric cancer and was treated with the ECX regimen but died after the first cycle of chemotherapy due to sepsis. The causes of deaths for all the TRDs were septicaemia (2), pneumonia (4), sepsis (4), renal failure (2), diarrhea (1) and unknown (1). The FN rate for the single agent capecitabine regimen was $1.6 \%(2 / 129)$ and the TRD rate was $5.4 \%$ (7/129). All the TRDs for the single agent capecitabine regimen occurred in situations where the drug was used for palliative intent.

\section{Discussion}

This study confirms the widespread acceptance of oral capecitabine into many chemotherapy regimens in clinical practice outside of clinical trials. Capecitabine was used in 14 different tumour sites in this study. As this study was from a cohort in 2009-2010, we assume its usage will be even more prevalent today. Its ease of administration compared to 5-FU has seen it being tested in many tumour sites where 5-FU has activity. This is especially relevant in institutions where there are insufficient oncology beds or it is costly for hospital admissions. Moreover, standard continuous 5-FU infusion is usually given for 4-5 days in head and neck cancers and gynaecological malignancies. Though this study did not have patients with these tumours using capecitabine, they are currently increasingly being used in our institution. Patients who are being treated with palliative intent for advanced or metastatic head and neck cancers have to be admitted for prolonged hospitalization when 5-FU is used. This may negatively impair their quality of life. With capecitabine, this problem is abrogated and in some situations no admission at all is needed when cisplatinum is replaced with carboplatin where platinum remains a backbone in the treatment of head and neck and gynaecological malignancies especially in the palliative setting.

The FN rate in this study is $2.2 \%$ for the overall population and $1.6 \%$ for patients who underwent capecitabine alone regimen. These rates are comparable to the published rates from randomized trials using capecitabine, ranging from $0.3 \%$ to $3.4 \%$ (Scheithauer et al., 2003; Twelves et al., 2005; Cassidy et al., 2008; Cassidy et al., 2011). These rates are better than the rates published for regimens using its counterpart, intravenous 5-FU. The risk of FN is higher in published studies with intravenous 5-FU based regimens ranging from 4\%-15\% depending on which regimen and also whether 5-FU is given in a bolus or continuous infusion fashion (Louvet et al., 2002; Rougier et al., 2003; Goldberg et al., 2004). Alas, capecitabine not only provides convenience it also reduces the need for admissions to treat FNs which often times may also be life-threatening.

The TRD rate in this study is $5.1 \%$ for the overall population and $5.4 \%$ for the capecitabine alone regimen. In a large randomized trial using the XELOX regimen for metastatic colorectal cancer, the TRD rate was $2.1 \%$ (Cassidy et al., 2008) while another Spanish trial reported a TRD rate of $0.6 \%$ (Diaz-Rubio et al., 2007). Single agent capecitabine was tested in the adjuvant setting for stage III colon cancer in the X-ACT trial and the TRD rate in this large trial was $0.3 \%$ (Scheithauer et al., 2003). The discordance between the TRD rates observed in our cohort and the randomized trials demonstrates the importance of determining the actual rates in routine clinical practice outside of trial settings. It is a norm for clinical trials to have strict inclusion and exclusion criteria including age limit, life expectancy of at least 3-6 months, exclusion of patients with poor performance status, significant co-morbidities and deranged biochemistry tests amongst others. However, in actual clinical practice clinicians may not have the option of excluding treatment based on these poor prognostic factors that may increase the risk of treatment related deaths. All but one TRD in our cohort of patients were treated with palliative intent. In such scenarios patients often has a significant burden of disease that would lower the patients' immunity and thus further compound the problem of immunosuppression with chemotherapy thus increasing the risk of TRD due to sepsis. However, palliative chemotherapy has been shown to be beneficial with survival benefit in many tumour sites including metastatic colorectal cancer which represents the majority of patients in this study cohort. It is in this light that we need to be cognizant of the fact that even though oral capecitabine is a convenient chemotherapy drug to use, it is no different from other intravenous chemotherapy agents with regards to risk of potentially life-threatening complications resulting in deaths. This is even more important in palliative setting when the treatment is not curative and the risks and side effects of treatment should be minimized. Patients and clinicians alike need to understand this risk before patients are able to give informed consent for chemotherapy. Patients' education regarding risk of chemotherapy is vital, so that they will seek medical attention early as to avoid clinical deterioration and death.

We acknowledge the shortcomings associated with a retrospective study though the endpoints of FN and TRD are non-ambiguous in nature. It would also have been better if the study concentrated on the most commonly used capecitabine regimens and more patients were recruited to give a better point estimate of the $\mathrm{FN}$ and TRD rates in actual clinical practice. In conclusion, we found that capecitabine is being used widely in clinical practice in a myriad of tumour sites and has a low risk of febrile neutropaenia. However, oral capecitabine is like any other intravenous chemotherapy agent, runs the risk of significant complications which may result in treatment related deaths. 


\section{References}

Bilici A, Selcukbiricik F, Demir, et al (2014). Modified docetaxel and cisplatin in combination with capecitabine (DCX) as a first-line treatment in HER2-negative advanced gastric cancer. Asian Pac J Cancer Prev, 15, 8661-6.

Bayoglu IV, Varol U, Yildiz I, et al (2014). Second-Line capecitabine and oxaliplatin combination for gemcitabineresistant advanced pancreatic cancer. Asian Pac J Cancer Prev, 15, 7119-23.

Cassidy J, Twelves C, Van Cutsem E, et al (2002). First-line oral capecitabine therapy in metastatic colorectal cancer: a favorable safety profile compared with intravenous 5-fluorouracil/leucovorin. Ann Oncol, 13, 566-75.

Cassidy J, Clarke S, Diaz-Rubio E, et al (2008). Randomized phase III study of capecitabine plus oxaliplatin compared with fluorouracil/folinic acid plus oxaliplatin as first-line therapy for metastatic colorectal cancer. J Clin Oncol, 26, 2006-12.

Cassidy J, Clarke S, Diaz-Rubio E, et al (2011). XELOX vs FOLFOX-4 as first-line therapy for metastatic colorectal cancer: NO16966 updated results. Br J Cancer, 105, 58-64.

Choi JG, Seo JH, Oh SC, et al (2012). A Phase II Trial of Gemcitabine plus Capecitabine for Patients with Advanced Pancreatic Cancer. Cancer Res Treat, 44, 127-32.

Chua DT, Sham JS, Au GK (2003). A phase II study of capecitabine in patients with recurrent and metastatic nasopharyngeal carcinoma pretreated with platinum-based chemotherapy. Oral Oncol, 39, 361.

Chua D, Wei WI, Sham JS, et al (2008). Capecitabine monotherapy for recurrent and metastatic nasopharyngeal cancer. Jpn J Clin Oncol, 38, 244-9.

Chua DT, Yiu HH, Seetalarom K, et al (2012). Phase II trial of capecitabine plus cisplatin as first-line therapy in patients with metastatic nasopharyngeal cancer. Head Neck, 34, 1225-30.

Chiu J, Tang V, Leung R, et al (2014). Efficacy and tolerability of adjuvant oral capecitabine plus intravenous oxaliplatin (XELOX) in Asian patients with colorectal cancer: 4-year analysis. Asian Pac J Cancer Prev, 14, 6585-90.

Ciuleanu E, Irimie A, Ciuleanu TE, et al (2008). Capecitabine as salvage treatment in relapsed nasopharyngeal carcinoma: a phase II study. J Buon, 13, 37-42.

Comella P, Massidda B, Filippelli G, et al (2009). Randomised trial comparing biweekly oxaliplatin plus oral capecitabine versus oxaliplatin plus i.v. bolus fluorouracil/leucovorin in metastatic colorectal cancer patients: results of the Southern Italy Cooperative Oncology study 0401. J Cancer Res Clin Oncol, 135, 217-26.

Cunningham D, Starling N, Rao S, et al (2008). Capecitabine and oxaliplatin for advanced esophagogastric cancer. $N$ Engl $J$ Med, 358, 36-46.

de Naurois J, Novitzky-Basso I, Gill MJ, et al (2010). Management of febrile neutropaenia: ESMO clinical practice guidelines. Ann Oncol, 21, 252-6.

Diaz-Rubio E, Tabernero J, Gomez-Espana A, et al (2007). Phase III study of capecitabine plus oxaliplatin compared with continuous-infusion fluorouracil plus oxaliplatin as firstline therapy in metastatic colorectal cancer: final report of the spanish cooperative group for the treatment of digestive tumors trial. J Clin Oncol, 25, 4224-30.

Ducreux M, Bennouna J, Hebbar M, et al (2011). Capecitabine plus oxaliplatin (XELOX) versus 5-fluorouracil/leucovorin plus ox-aliplatin (FOLFOX-6) as first-line treatment for metastatic colorectal cancer. Int J Cancer, 128, 682-90.

Goldberg RM, Sargent DJ, Morton RF, et al (2004). A randomized controlled trial of fluorouracil plus leucovorin, irinotecan, and oxaliplatin combinations in patients with previously untreated metastatic colorectal cancer. J Clin Oncol, 22, 23-30.

Jenkins AD, Ramondetta LM, Sun C, et al (2005). Phase II trial of capecitabine in recurrent squamous cell carcinoma of the cervix. Gynecol Oncol, 97, 840-4.

Kang YK, Kang WK, Shin DB, et al (2009). Capecitabine/ cisplatin versus 5-fluorouracil/cisplatin as first-line therapy in patients with advanced gastric cancer: a randomised phase III noninferiority trial. Ann Oncol, 20, 666-73.

Li YH, Wang FH, Jiang WQ, et al (2008). Phase II study of capecitabine and cisplatin combination as firstline chemotherapy in Chinese patients with metastatic nasopharyngeal carcinoma. Cancer Chemother Pharmacol, 62, 539-44.

Louvet C, Andre T, Tigaud JM, et al (2002). Phase II study of oxaliplatin, fluorouracil, and folinic acid in locally advanced or metastatic gastric cancer patients. J Clin Oncol, 20, 4543-8.

Martinez-Trufero J, Isla D, Adansa JC, et al (2010). Phase II study of capecitabine as palliative treatment for patients with recurrent and metastatic squamous head and neck cancer after previous platinum-based treatment. Br J Cancer, $102,1687$.

Miwa M, Ura M, Nishida M et al (1998). Design of a novel oral fluoropyrimidine carbamate, capecitabine, which generates 5 -fluorouracil selectively in tumours by enzymes concentrated in human liver and cancer tissue. Eur J Cancer, 34, 1274-81.

Okines AF, Norman AR, McCloud P, et al (2009). Meta-analysis of the REAL-2 and ML17032 trials: evaluating capecitabinebased combination chemotherapy and infused 5-fluorouracilbased combination chemotherapy for the treatment of advanced oesophago-gastric cancer. Ann Oncol, 20, 1529-34.

Rougier P, Mitry E, Cunningham D, et al (2003). Is age a prognostic factor of toxicity and efficacy in patients (pts) with metastatic colorectal cancer (MCRC) receiving irinotecan in combination with 5FU/folinic acid (FA). Proc Am Soc Clin Oncol, 22, 267

Scheithauer W, Mckendrick, Begbie S, et al (2003). Oral capecitabine as an alternative to i.v. 5-fluorouracil-based adjuvant therapy for colon cancer: safety results of a randomized, phase III trial. Ann Oncol, 14, 1735-43.

Stockler MR, Harvey VJ, Francis PA, et al (2011). Capecitabine versus classical cyclophosphamide, methotrexate, and fluorouracil as first-line chemotherapy for advanced breast cancer. J Clin Oncol, 29, 4498-504.

Sym SJ, Chang HM, Ryu MH, et al (2010). Neoadjuvant docetaxel, capecitabine and cisplatin (DXP) in patients with unresectable locally advanced or metastatic gastric cancer. Ann Surg Oncol, 17, 1024-32.

Twelves C, Wong A, Nowacki PN et al (2005). Capecitabine as adjuvant treatment for stage III colon cancer. $N$ Engl $J$ Med, 352, 2696-704.

Van Cutsem E, Twelves C, Cassidy J, et al (2001). Oral capecitabine compared with intravenous fluorouracil plus leucovorin in patients with metastatic colorectal cancer: results of a large phase III study. J Clin Oncol, 19, 4097-106.

Van Cutsem E, Hoff PM, Harper P, et al (2004). Oral capecitabine vs intravenous 5-fluorouracil and leucovorin: integrated efficacy data and novel analyses from two large, randomised, phase III trials. Br J Cancer, 90, 1190-7.

Wadell TS, Chau I, Barbachano Y, et al (2012). A randomized multicenter trial of epirubicin, oxaliplatin, and capecitabine (EOC) plus panitumumab in advanced esophagogastric cancer (REAL3). J Clin Oncol, 30, 18.

Varol U, Dirican A, Yildiz I, et al (2014). First-line mono- 
Capecitabine Pattern of Use, Febrile Neutropaenia and Treatment Related Death in Asian Cancer Patients chemotherapy in frail elderly patients with metastatic colorectal cancer. Asian Pac J Cancer Prev, 15, 3157-61.

Vasey PA, McMahon L, Paul J, et al (2003). A phase II trial of capecitabine $\left(\right.$ Xeloda $\left.^{\circledR}\right)$ in recurrent ovarian cancer. British J Cancer, 89, 1843-8.

Xiong HQ, Varadhachary GR, Blais JC, et al (2008). Phase 2 trial of oxaliplatin plus capecitabine (XELOX) as secondline therapy for patients with advanced pancreatic cancer. Cancer, 113, 2046-52.

Yoney A, Isikli L (2013). Can capecitabine be used instead of concurrent bolus 5-FU in postoperative chemoradiotherapy for gastric adenocarcinoma? Asian Pac J Cancer Prev, 14, 5127-31. 